the additive model. The work was conducted under project NS 10596 of the Czech Ministry of Health.

\title{
P71 LUNG CANCER RISK FROM OCCUPATIONAL AND ENVIRONMENTAL RADON AND ROLE OF SMOKING
}

Ladislav Tomasek National Radiation Protection Institute, Prague, Czech Republic

10.1136/oemed-2011-100382.285

\section{Objectives and}

Methods The study is based on two large Czech cohorts of uranium miners and people exposed to high levels of radon in houses with 1107 and 240 cases of lung cancer. Individual smoking data were collected for most of subjects (68\% and $75 \%$ ). The risk is evaluated in dependence on cumulated exposure with effect modification by time since exposure and smoking. Results and conclusions It is shown that the excess relative risk per unit exposure from earlier periods of exposure is about $1 / 3$ in comparison to recent exposures, whereas the relative effect from exposures before 35 years is only about $1 / 10$. Smoking specific estimates of excess relative risk per unit exposure were 3-5 times higher among never smokers in both studies. The interaction of radon and smoking is evaluated by geometric mixture models and is found to be between additive and multiplicative model. When smoking specific equivalent doses to the lungs and modifying effect of time since exposure are used, the combined effect of radon and smoking is close to 